\title{
COMPLEXITY SCIENCE AS AN ASPECT OF THE COMPLEXITY OF SCIENCE
}

\author{
DONALD C. MIKULECKY \\ Senior Fellow, Virginia Commonwealth University's Center for the Study of \\ Biological Complexity, Richmond, VA
}

\section{INTRODUCTION}

How can we treat science as an object of scientific inquiry? The central problem arises with that question. Science has tried to rid itself of circularity and in so doing has become a very limited method for examining the complex world it tries to have us understand. Self reference is at the center of so many of the interesting thing we want to understand including and especially life itself. The existence of this self referential character is the essence of what we have come to call "complexity". The works of Robert Rosen [1, 2, 3] spell this out in great detail. This series of investigations began over a half century ago yet still remains virtually unrecognized by the vast majority of those who call themselves "scientists". That fact alone can be a springboard to launch a study of science as an object, which is what this study is all about. I have reviewed the technical aspects of Rosen's work elsewhere [4] and will consider the broader philosophical implications here.

Using the ideas Rosen developed, we can begin with the following working definition of complexity:

Complexity is the property of a real world system that is manifest in the inability of any one formalism being adequate to capture all its properties. It requires that we find distinctly different ways of interacting with systems. Distinctly different in the sense that when we make successful models, the formal systems needed to describe each distinct aspect are NOT derivable from each other.

Rosen created a dichotomy between complex system and simple systems, or mechanisms. The essence of that dichotomy is summarized in Table 1. 
Table 1. Complex systems vs. simple mechanisms.

\section{Complex}

No largest model

Whole more than sum of parts

Causal relations rich and intertwined

Generic

Analytic Synthetic

Non-fragmentable

Non-computable

Real world

\author{
Simple \\ Largest model \\ Whole is sum of parts \\ Causal relations distinct \\ Non-generic \\ Analytic $=$ Synthetic \\ Fragmentable \\ Computable \\ Formal system
}

This set of properties that distinguish disjoint categories of objects is the subject of Rosen's life work. Any brief summary will be lacking the content developed to establish them in detail, but can give a sense of what the categorization entails.

The categories are disjoint because of a central characteristic of the way we go about practicing science. We create a surrogate world, the world of simple systems, to enable our minds to try to grasp the complexity of the world we wish to comprehend. Hence the real world is complex and must be reduced to something less than its many faceted reality in order to be studied. The surrogate simple world is a world of formal systems or models, that replace the many faceted complex reality with something our minds can work with. Our understanding depends on this process. The process has consequences that can not be escaped. The table of characteristics is a summary of those consequences, and they will be discussed briefly.

\subsection{The largest Model}

The formal system science has traditionally used to model the complex world has a largest model. This model is the crux of scientific description and can be called the "Newtonian Paradigm" using terminology adopted by Rosen. The Newtonian Paradigm is a world constructed in terms of non-linear dynamics in its many forms. The most modern forms all resolve to the largest model formulated as non-linear dynamics. Included are chaos theory, cellular automata, and non-linear systems of partial differential equations or state space descriptions, among others. These formalisms lend themselves well to the use of modern computing methods and therefore computability becomes a hallmark of the largest model used to represent 
simple systems. Real systems go beyond computable properties and can only be approximated by computer simulation. This has been said in many ways, such as "The map is not the territory", and will often be acknowledged in specific instances, only to be denied vigorously when proposed as a general truth. In the working definition of complexity, this is manifest as the need for distinct formalisms in order to completely represent complex reality. In fact, there is no finite number of such descriptions.

\subsection{Why is the whole more than the sum of its parts?}

A mantra in complexity research is the notion that any complex whole is more than the sum of its parts. The world of simple systems created by science does not escape this idea. We will give some examples of models that can demonstrate that the same material parts can be assembled in different ways to make very different functioning wholes. The key word being "functional" for it is in function that this property of complex reality is rooted. More interesting is the recognition that function is as much an ontological aspect of complex reality as are the atomic and molecular material building blocks. This must be so if the notion that the whole is more than the sum of its parts is to have any meaning. Reducing a complex whole to smaller units and eventually to atoms and molecules destroys function and destroys the very identity of the complex whole. This thing called "function" that characterizes complex wholes is as real as the material aspects of the system. Simple systems can be reduced to their parts and are called machines or mechanisms for this reason. This leads to an unfortunate ambiguity between different meanings of the word "machine". An automobile is a man made machine and can seem to be identical with the formal description used to create it. However, the real world automobile is a complex entity only approximated by its formal description even though it can be built from that formal description. It will be important to keep the vernacular meaning of machine, the complex reality, separated from its formal description as a machine or simple mechanism. In the relational systems theory being developed as an approach to complex system description using the ideas introduced by Rosen, the material description is sacrificed in favor of a functional description. The complex whole is reduced to a combination of functional components that can not be mapped to material parts on a one to one basis. These functional components only have definition in the context of the complex whole and are destroyed when it is reduced to material parts. Hence the functional components are the 
way that the things that are "more than the sum of the material parts" become entities having an ontology of their own. This idea is central to an understanding of complexity.

\subsection{Causality and information: Science of method and science of content}

Science has often been characterized by its methodology. This has been the key feature that has kept science from being "contaminated" by the logical problems that are inherent in things like circularity and self reference. The limits imposed by this methodology also keep science from dealing adequately with complex reality for this very reason. Within science a very limited definition of "information" was developed by Shannon and Weaver to provide a tool in communications theory. The shortcomings of this very sterile definition show up again and again because it does not satisfy the intuitive notion if what the term "information" connotes. This fact is closely linked to the relationship between syntax and semantics. The concept of information they formulated deals only with syntax while what is really needed is a way of also dealing with semantic content. Rosen introduced another kind of information. The difference between the two versions of information is related to the complexity/simple mechanism/machine dichotomy. Science, by dealing with mechanisms, led to questions about "how" things work. The other aspect to information deals with "why" things are as they are. The question "how does it work?" is a reductionist/mechanist question. The strict methodology of hard reductionist science does not allow "Why?" questions because the method is impotent to deal with them. Yet there is much information content in the answer to the question "Why?" It was Aristotle that taught us that. Aristotle gave four answers to the question "Why?" These causes or "becauses" are the material, efficient, formal, and final causes. They are often illustrated as the answers to the question "Why a house?"

Material cause: Bricks, wood, metal, glass, and other materials that make up the house.

Efficient cause: The builders that put those materials into the form of a house giving it a function beyond that of the unassembled materials.

Formal cause: The plan or blueprint used to give the house its form as a house.

Final cause: The purpose or function of the house, to be a dwelling place. 
In machines these causes can always be separated. In real complex systems they are entwined and not capable of being separated as in a machine. The example to follow, the distinction between an organism and a machine, will establish this in a clear example.

\subsection{Which is generic, physics or biology?}

The widely accepted myth that biology is special and that physics is generic is totally wrong. In fact, far more can be learned about the material world by a careful study of biology than can ever be learned from physics. Physics is the model of hard reductionist science. Physics envy has done real damage to fields that want to be considered "scientific" such as psychology and sociology among others. Biological and social systems are clearly complex only because they have resisted the attempt to find an acceptable largest model as has been done in physics.

\subsection{Analytic vs. synthetic models}

The distinction between analytic and synthetic models is a mathematical one. It is based on the use of direct products and direct sums as the way the models are constructed. The definition of these structures comes from category theory. Rosen developed his own form of category theory but it does not differ that much from other versions. Category theory is that branch of mathematics created to overcome some problems in set theory. It is actually a way of modeling mathematics itself. To summarize the essential difference between analytic and synthetic models is a matter of looking at the way set like entities can be combined. Product sets are based on a set theoretical operation called a Cartesian product. The Cartesian product of a pair of sets $\mathrm{A}=(\mathrm{a} 1, \mathrm{a} 2, \mathrm{a} 3)$ and $\mathrm{B}=(\mathrm{b} 1, \mathrm{~b} 2)$ is symbolized as $\mathrm{A} \mathrm{x}$ $\mathrm{B}$ and consists of the collection of pairs (a1, b1), (a1, b2), (a2, b1), (a2, $\mathrm{b} 2),(\mathrm{a} 3, \mathrm{~b} 1),(\mathrm{a} 3, \mathrm{~b} 2)$. The direct sum of these entities is merely the combination of the members of A and B. In simple systems the direct product can always be reduced to the direct sum. They are equivalent. This is simply a mathematical way of describing the reducibility or fragmentability of reductionist models. In the case of complex systems, this is never true. There is more involved because complex systems are modeled in terms of their functional components and these functional components consist of direct products that are not capable of being reduced to parts without the destruction of the functional component. 


\subsection{Fragmentability}

It follows directly from what has been developed so far that the complex system with its context dependent functional components can not be fragmented into material parts. Simple mechanisms or machines can always be fragmented into material parts.

\subsection{Computability}

This subject can fill a number of books. It is the subject of heated debates and for those who have placed their faith in computers and computable models, the stakes are very high. The sides of this debate can be exemplified on one hand by the hard core proponents of "artificial life" and on the other hand by Robert Rosen and many others who have come to see his understanding of the complex real world as a fundamental breakthrough in world models. The proponents of the reductionist/mechanistic view believe that the Church-Turing thesis is correct.

\section{SCIENCE AS A COMPLEX SYSTEM}

Science is a complex system. It has many facets and many different ways of being defined, described and analyzed. The most obvious use of the word is as a noun. What does the noun stand for? It may stand for an ideal that we sometimes call "pure" science. Such ideals are approximated in reality and never really realized completely. Science as a noun also stands for a body of knowledge. Science is also a set of human institutions. Science can also mean those things that the people we recognize as scientists do. When we speak of science in that way we can also speak of an activity and we sometimes refer to doing science so there is a verb form involved. We often describe things as scientific as in a scientific study or a scientific expedition. Then we see that there are adverbs and adjectives as well. There is also the scientific method. There is a philosophy of science. This philosophy is very important because it is the only way we can complete the system. That is, if we were forced to study science using science we would be caught in an impredicative self referential loop. This may be a partial explanation of why scientists seem to avoid philosophy, hoping to escape the need for another approach..

Science as a human institution also has a very important set of political facets. The products of scientific activity are often a source of wealth and power. The doing of science is an expensive enterprise and it must have 
its patrons. Both complexity theory and the philosophy of science run into this political aspect of science in a number of ways. By describing the limits of the reductionist/mechanist paradigm there is a danger of casting doubt on the ability of science to produce what its patrons expect from it. Teaching such things to science students can place them in situations that are difficult. Students must choose projects to satisfy their mentors who in turn must satisfy their patrons. Reflecting these strong shaping forces back on the definition we so roughly outlined, it should be clear that differences between science as an ideal and science as what is practiced by scientists can be very great if the ideal is involved with showing limits rather than convincing patrons that their investment will pay off.

As the research tools and equipment grow in expense and sophistication, the tendency to occupy one's time using the equipment also grows. Thus the activity we call science becomes driven by questions that can be answered by the equipment rather than the desire to know in its "purest" form.

The methodology of science is not codified in any clear way. Books have been written about the best way to accept candidates for scientific law and scientific theory. Books have also been written about the failure of other disciplines to satisfy the criteria of the scientific method.

Periodically there are so called paradigm shifts claimed. Often these are not universally recognized as such, but what ever name one wants to assign, change does occur in the method and this can be significant in its magnitude. Proponents of complexity theory in its many forms often ask for it to be a candidate for such status.

Is there a model for this kind of complexity in systems of human thought and the activities that feed in to such systems? Clearly there is. Robert Rosen chose Number Theory as his example. He reviewed the efforts of the formalists to purge the field of all circularities and self referential loops. To make a very long story very short, the attempt failed miserably. Each attempt to present a finished product was met by demonstrations that important things about numbers were left out. This was because they were insisting that the theory be self consistent and rightly so. It took Kurt Gödel to prove that such systems can not be both self consistent and complete. By requiring self consistency they doomed their efforts to produce an incomplete system. The only way to deal with complex systems was to rely on something external to attempt the completion. But then the larger system is subject to the same problem and an infinite regression results. This issue has come up often in discussions among complexity theory proponents. One way some seem to satisfy themselves that they need not heed 
the problem is by claiming that Number Theory is mathematics and not science and that Gödel's proof has not been shown to apply to physical systems. This seems to be a false solution to the dilemma, especially since all the models science uses are mathematical

\section{COMPLEXITY AS AN ATTRIBUTE OF NATURE}

The use of the term "complexity" has come to be commonplace both in the scientific literature as well as in the lay press. A complex idea in its own right, complexity presents a challenge to those who would define it or codify its contents. Why is "What is complexity" a question not so easily answered? Universities and other research institutions have programs in "complexity research" and journals carry this word in their title. What is complexity? What does it mean to be "complex"? The dictionary does not help us here. There is more to the idea than what a dictionary definition suggests.

We need to look within science, where words are carefully defined and have more precise meanings, since it is here where the answer does lie. The concept of complexity that is of so widespread interest was born within the confines of the scientific community, had to struggle for existence, and finally grew into a substantial force in scientific thinking. The answer lies in the nature of science. Science helps us to understand the world around us by creating a surrogate world that is understandable. The real world is where complexity has its home. The world science gives us has become simple and understandable.

\subsection{Hard Science is built on Cartesian Reductionism}

Hard science can be best-defined in terms of Cartesian Reductionism and the machine metaphor. Descartes gave us the machine metaphor and he did it in a very interesting way. In the case of living systems he saw the body as a biological machine and the mind as something apart from the body. This is called Cartesian Dualism and survives to this day as one approach to the so-called mind/body problem. What the machine metaphor did was to set the tone for modern science. It is still with us. Descartes really did not know what a machine is, or if he did, he never told anyone. Ironically, not only do we not have a good definition of complexity, but we also lack one for a machine. The duality between these concepts is the key to the resolution of the problem. The importance of the machine metaphor is in the intuitive concept of machine that almost everyone shares. A machine is 
built up from distinct parts and can be reduced to those parts without losing its machine-like character. We call this idea "Cartesian Reductionism". We have seen that complex (real) systems can not be successfully reduced to material parts without the loss of some significant attributes in the process. This led to the axiom that the whole is more than the mere sum of its parts. Adopting this axiom as a truth leads to a conclusion that is inescapable. If the whole is more than the sum of its parts there must be more to things than atoms and molecules. Reducing a complex whole in the real world always destroys something. What is lost is an elusive entity, but is also the central idea in the concept of complexity as applied to the real world. If there is more to things in the real world than atoms and molecules then this something that is there has an existence, an ontology. This is the reason why as a concept complexity is a difficult one. It can not be both ways. Either a real world whole is more than the sum of its parts or it can be reduced to atoms and molecules with nothing being lost and the world is made of machine-like things. Cartesian reductionism does not work for making models of complex systems; it only reduces them to simple mechanisms that may reflect some aspect, but merely as a shadow of the complex whole

\subsection{The Newtonian paradigm is the modern manifestation of hard science}

Newton gave us, among other things, three laws of motion, which were intended to describe the motion of the planets. It turned out that methodology spawned by the application of these laws could be applied in a seemingly perfectly general way. This broader application has been the foundation of the modern scientific method and will be referred to here as the Newtonian Paradigm.

In the center of this paradigm is dynamics. Dynamics is the way the laws of motion get applied. The local description of the motion is formulated as a differential equation called an equation of motion. The equation of motion is manipulated by using the calculus (integrated) and results in a trajectory, which is an algebraic equation for calculating a particle's position as a function of time. Later, this was made somewhat more complicated by quantum mechanics, but the central philosophy has never changed.

The paradigm has been generalized from particle motion to all systems if we recognize that quantum mechanics is part of that generalization. The world-view called reductionism has as a central theme that anything in 
the material world can be reduced to particle motion, that is, the motions and interactions of atoms and molecules. When we look carefully at the subject matter of physics, we see that it is the application of the Newtonian Paradigm to the universe. This application then makes the world into simple mechanisms. That is to say that the subject matter of physics is the study of simple mechanisms. Note that in this context, "simple" means the opposite of complex, not the opposite of complicated.

\subsection{Complexity is the result of the failure of the Newtonian Paradigm to be generic}

A careful look at the real world tells us that it is complex. Yet, the success of the Newtonian Paradigm cannot be ignored. Most of modern science and technology is the result of it. For that reason alone it is difficult to suggest that it has limits and to then make that suggestion stick. Not only does the paradigm have limits, but also those limits are what gave rise to a concept like complexity.

\subsection{The way science is done: The modeling relation}

How is science done? It is a combination of using our senses to observe the world around us and then to use some mental activity to make sense out of that sensory information. The process is what we will call the modeling relation. If we call the world we are observing and/or trying to understand the Natural System and the events that make it change as we observe causality, then that represents our object of study. What we do in our minds is to encode the natural system into another system that is of our making or choosing which we can call a formal system. Once we have chosen a formal system, we can manipulate it in various ways with the objective of mimicking the causal change in the natural system. These manipulative changes in the formal system we will call implication. Finally, once we think we have an appropriate formal system and have found an implication that corresponds to the causal event in nature, we must decode from the formal system in order to check its success or failure in representing the causal event. Figure 1 represents the modeling relation we have just described.

If all the parts of the diagram are in harmony, in other words if $1=2+$ $3+4$, we say that the diagram commutes and we have a model. A model of the world is the outcome of a successful application of the scientific method, but it can also arise in other, less formal ways. Whenever someone tries to make sense out of the world, they are trying to construct a successful 


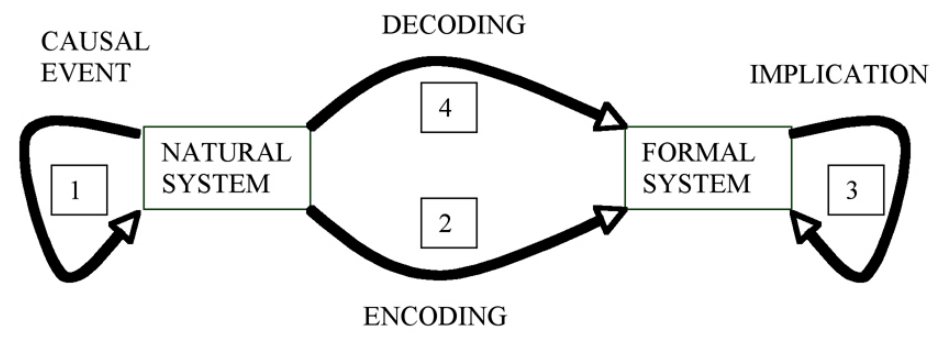

Figure 1.

modeling relation, or a model.

\subsection{Complex systems and simple systems are disjoint categories that are related by the modeling relation}

The world therefore divides naturally into those things that are simple and those things that are complex. The real world is made up of complex things. It is something we encounter constantly and try to make sense of. The way we do that is to encode it into formal systems. Therefore the world of simple mechanisms is a fictitious world created by science or, more specifically, by physics as the hard version of science as a formal system which hopes to model the real world. This is the world of the reductionist. It is modeled by the Newtonian Paradigm and simply needs sufficient experimentation to make it known to us. Those experiments involve reducing the system to its parts and then studying those parts in a context formulated according to dynamics.

Therein lays the reason for the restrictions on the scientific method. The method involves a rigid empiricism that feeds data into the formal system. Here is the philosophical rub. The epistemology spills over into an ontology. The modeling relation is forgotten (but it is always there) and the formal system has become reality.

Now the definition of complexity is complete. The world, from which we single out some smaller part, the natural system, is converted into a formal system that our mind can manipulate and we have a model. The world is complex. The formal system we chose to try to capture it can only be partially successful. For years we were satisfied with the Newtonian Paradigm as the formal system, forgot about there even being and encoding and decoding, and gradually began to change the ontology so that the Newtonian Paradigm actually replaced or became the real world (at least 
as seen through the eyes of science). As we began to look more deeply into the world we came up with aspects that the Newtonian Paradigm failed to capture. Then we needed an explanation. Complexity was born! This easily can be formalized. It has very profound meaning.

\section{THERMODYNAMIC REASONING AS A TRANSITION TO COMPLEXITY SCIENCE}

Rosen had little to say about thermodynamics in his critique of reductionism in the form of the Newtonian paradigm. There is probably a good reason for this. Clifford Truesdell [5] once made a very good case for thermodynamics having a certain "strangeness" as a part of physics, or we could say as part of the reductionist Newtonian paradigm. This strangeness needs to be considered in more detail for it reveals the seeds of the ideas Rosen found to be true about the mechanistic approach to reality. Thermodynamics is probably more poorly understood by mechanistic scientists than any other branch of physics. The reasons for this are deep and revealing.

One of the most serious consequences of the inability of those doing either mechanistic science or thermodynamics to see the problem clearly is the resultant gap in the Newtonian largest model. It is possible to see mechanistic reasoning and thermodynamic reason as different models of complex reality in the spirit of the ideas discussed here. Instead, from its beginnings thermodynamics was put under severe pressure to conform to the mechanist's largest model and thereby suffered a lack of development as its own alternative to mechanistic physics.

Thermodynamics came into being for very practical reasons. The boring of cannons, the brewing of beer and the steam engine are but a few of the reasons that the frictionless world of Newton's paradigm needed to be patched up. Heat as a form of energy also had to be dealt with. Perpetual motion machines had to be dealt with in a rational manner to curtail the squandering of time and energy, sometimes by very bright scientists. As progress was made, there were also immediate problems presented to the those who had been content with the frictionless world where heat was merely another form of energy and nothing more troublesome than that. Since the material world was to be understood in mechanistic terms, a new kind of mechanics called "statistical" mechanics had to be developed to try to make a bridge between thermodynamic reasoning and mechanistic reasoning.

Why is this so? The answer is one of the best demonstrations of the 
power of Robert Rosen's analysis even though he may not have ever seen it himself. There is a fundamental difference between mechanistic reasoning and thermodynamic reasoning and it can not be erased by the limited successes of the use of statistical mechanics to bring them together.

Thermodynamics is about those properties of systems that are true independent of their mechanism. This is why there is a fundamental asymmetry in the relationship between mechanistic descriptions of systems and thermodynamic descriptions of systems. From the mechanistic information we can deduce all the thermodynamic properties of that system. However, given only thermodynamic information we can deduce nothing about mechanism. This is in spite of the fact that thermodynamics makes it possible for us to reject classes of models such as perpetual motion machines. (This does not stop such models from appearing in subtle forms in the modern literature.) This asymmetry is poorly understood because thermodynamics is not a tool mechanists see as valuable. Some of this attitude is justified by the structure of thermodynamics as it exists in texts and courses. In some ways the field is a prisoner of its own history. It is useful to examine that history with this problem in mind.

\subsection{Classical or "equilibrium" thermodynamics and its limits}

Thermodynamics recognizes distinct systems categories based on the constraints imposed on the system in order to study it systematically. This thought process is very much like the defining of a "universe of discourse" in other fields. A system is defined in terms of the way we allow it to interact with the outside world. Often it is useful to see these interactions as having to occur across some real or imaginary boundary or "skin" surrounding the system. There are only three possible categories based on this mode of reasoning:

(1) The isolated system. Isolated systems are isolated from the rest of the world by a boundary that is impermeable to both energy and matter flow. Such systems define the notion of "equilibrium", in the thermodynamic sense, as the state the system is in after a sufficient amount of time is allowed to pass. The equilibrium state is characterized by the disappearance of the "transient" flows of matter and energy within the system that brought it to this final state wherein nothing further can happen. That is true in the large. Very locally, fluctuations around this equilibrium point are 
happening as a manifestation of the thermodynamic property called "temperature". The fluctuations are atomic and molecular motion and increase in magnitude as heat energy is added to the system and decrease in magnitude as it is removed. Isolating the system will result in a system at constant temperature consistent with its heat content.

(2) Closed systems are systems with boundaries that will allow the transfer of heat energy through them but these boundaries are impermeable to matter flow. Thus closed systems contain a fixed amount of material but can be warmed or cooled by manipulating their heat content. Such systems are capable of being put in contact with reservoirs of heat energy at different temperature to induce a flow of heat through them and are therefore capable of being maintained in stationary states away from equilibrium.

(3) Open systems. These systems can exchange matter and heat energy with their environment and need never reach an equilibrium. These systems can exist in stationary states, periodic states, as well as chaotic states.

The laws and ideas of thermodynamics were developed with great care by doing thought experiments on equilibrium systems. Virtual "quasistationary processes were conceived by imagining so called "infinitesimal" changes to take the isolated system from one equilibrium state to another. Sadi Carnot developed a clever method of bringing the system through a cycle so that it always ended in its starting equilibrium state.

One central characteristic of equilibrium is the existence of a thermodynamic variable called "entropy", S, defined as the quotient of the heat energy in the system, Q, and absolute temperature of the system, T:

$$
S=Q / T
$$

More useful was the notion of the entropy change associated with an isothermal quasi-stationary process:

$$
d S=d Q / T
$$

The entropy of a system, to oversimplify somewhat, is a measure of the "quality" of the energy of that same system. This follows from reasoning about heat engines being operated in cycles. In order for a heat engine (e.g. a steam turbine) to produce work that can be used it must have a source of 
hot steam and that steam must flow through it to a reservoir that is cooler. The First law of thermodynamics is nothing new to physics since it is simply the idea that energy can not be created or destroyed. Hence a stem engine uses some of the heat energy, converts it to mechanical work, another form of energy, and allows the rest of the heat energy to pass through it with the matter (in this case water and/or water vapor) that is conserved in the process. The Second law of thermodynamics forbids the complete transfer of heat energy to mechanical work as well as forbidding the operation of the engine between a source and sink at the same temperatures. This is why the entropy became a useful and necessary concept. The result of "cooling" the matter in the process of extracting mechanical work, increased the heat/ absolute temperature quotient or the entropy of the system. One way of stating the second law is that there is a requirement that any real process must result in the increase of entropy overall. It is possible to make devices that locally decrease entropy, but only if the global result is an increase.

At equilibrium, there is no change in the amount of entropy.

$$
d S=0
$$

In any real process,

$$
d S>0
$$

The fact that so much of what we know about thermodynamics came from reasoning involving equilibrium and isolated systems is ironic when we recall that it was practical matters, the doing of things, the carrying out of processes that motivated the entire development. Nevertheless, by resorting to what now seems to be a rather clumsy thought process involving the carrying out of processes by performing small incremental changes, much was learned.

What is problematic is that the world of real processes is what is of interest and equilibrium are the natural endpoints of some of those processes in the situation where the system is totally isolated.

\subsection{Dissipation, friction, and irreversibility}

Energy is conserved, but entropy must increase in the carrying out of any process. The first two laws of thermodynamics are imbedded in that sentence. The statement is true of all systems regardless of mechanism. The result of this is that physics has two realizations. The original physics of 
Newton and his followers was the physics of systems that had dynamics yet were without the creation of entropy. The "ideal" pendulum, for example, can not exist and is the end of a limiting process where friction is diminished to zero. Friction is another manifestation of the second law of thermodynamics. It is the recognition that in any real process some of the energy must be converted to heat energy. This heat energy can not be converted back to the original form of energy by the same process. The process is "irreversible". One good way to see this is in a famous experiment performed by Joule to measure the factor that must be used when energy in the form of mechanical work is changed to heat energy by any process. The number he obtained was called the mechanical equivalent of heat. The device he used has value as a source of insight far beyond its utility in obtaining that number.

The device was a jar of water surrounded by an insulating jacket having the purpose of isolating the system thermally. Thus heat energy could not enter or leave the system through its walls. At the top of the jar was mounted a well insulated crank attached to a paddle wheel inside the jar. Turning the crank allowed mechanical work to be performed on the water in the jar. The system allows a simple mechanistic explanation for how the mechanical work is converted to heat energy. The water is put into motion in a directed manner by the paddle wheels, but looses that directed motion eventually, the motion rather becoming "random" motion characteristic of heat energy. Having a thermometer mounted so that the water temperature can be monitored allows the direct relation between the work done turning the crank and the appearance of heat in the jar, using the heat capacity of water to calculate the amount of added heat energy from the increase in measured temperature.

The process is irreversible. There is no way to reverse this randomization of molecular motion to turn the paddle wheel and thereby gain back the mechanical work. The energy has been converted once and for all as long as it is confined to the thermally isolated jar.

This is a very revealing example of the nature of friction. Sometimes it is difficult to see a mechanistic picture of how this frictional "dissipation" of energy occurs. Chemical reactions have their own version of friction and are as irreversible as any mechanical process. In electricity the resistance to the flow of electrons results in electrical energy being converted to heat.

Knowing this, physics has created two categories for the systems it studies, conservative and non-conservative systems. Newtonian dynamics was developed using conservative, frictionless systems (fictional) and then ex- 
tended to the more difficult non-conservative systems that, as real world processes, involved friction necessarily. These non-conservative systems were irreversible and needed to be open if they were to do anything other than wind down to an equilibrium state and stop.

\subsection{Preserving the paradigm involved considering friction, irreversibility and dissipation}

It wasn't until the work of Onsager [6, 7] and others in the 1930's that a systematic physics of non-equilibrium, irreversible systems was developed. This new physics was thermodynamic rather than mechanistic, but the field of statistical mechanics was later extended to the non-equilibrium domain making the problem of backing up the thermodynamic knowledge with mechanical interpretations in terms of atomic and molecular motion very much more difficult. The increase in difficulty has severely limited the scope of these endeavors. Onsager and the others developed a thermodynamic approach and escaped most of that difficulty. They did so in a way that had its own inbuilt limitations, but that would not be seen for about forty more years when Network Thermodynamics was developed by Oster, Perelson, and Katchalsky and independently by Peusner [8]. The approach Onsager and the others introduced is am "phenomenological" approach. This is very different from the mechanistic physics that was used to describe nonequilibrium dynamic systems up to that time. Onsager's work was part of what won him a Nobel Prize. Later, Prigogine won a Nobel prize for building on the work of Onsager. One can speculate that Katchalsky may have been there in Prigogine's place had he not been gunned down by terrorists in 1972. That event changed the history of science and the history of the world. It was Kedem and Katchalsky who introduced non-equilibrium thermodynamics to biology and changed the way we dealt with all the dynamic processes occurring in living systems. Then, along with Oster and Perelson, he explored the new approach that married non-equilibrium thermodynamics to the huge body of theory generated for dynamic electric networks.

The impact of all this and the work of others that built on it is largely not understood by many even after complexity theory has grown in its impact on science. The reasons are many and not easily identified without being critical of the way science is practiced. The urge to preserve the mechanistic paradigm and to see thermodynamics as only an appendage has been too strong and in that way too destructive. When quantum theory was born it 
also was shaped by the same urges. The difference is that quantum theory is so much more adaptable to the mechanistic reductionist approach. Its mathematics and the interpretation of the mathematics could be given the very same form as what it was clearly showing to be a failure of Newtonian mechanics. Thermodynamics, on the other hand, uses a different form of mathematics. To most scientists satisfaction quantum mechanics simply helped further the knowledge generated by the Newtonian paradigm and did it little harm. It allowed the reductionist philosophy to appear to have established itself at all levels in the material description of reality. It became possible to see a more or less universal bottoms up approach to science. Yet there are findings from network thermodynamics that raise some very interesting, and possibly troubling questions about this. The lack of interest in these questions parallels a similar lack of interest in questions raised about science by Robert Rosen as he explored the complexity of the world science seemed to have mastered. It is very important that these questions and the findings that have been generated by the use of thermodynamic reasoning that is not constrained by the mechanistic mind set do not get totally ignored and forgotten Yet that may be exactly what is happening as reductionism forges ahead. Some insights into these events of scientific history and the philosophical oversight or lack of same can be had thanks to the contributions of Lakoff [9] who has applied cognitive linguistics to similar situations in politics. His ideas can be married to a concept of memes as "packages" of ideas that get passed along in a manner much analogous to genes.

\subsection{Framing the question in science:" Don't think about the whole system"}

As we make models of the world we necessarily use what we have already put together in our minds. The idea that complete objectivity is possible arises when the nature of the modeling relation is misunderstood. A model has many components. It consists of a number of subjective choices. The choice of the formal system we use to encode sensory information in order to think about how the real world is actually operating is a subjective choice. The illusion of objectivity arises from the fact that the question has been framed in a particular way. The idea that there is only one encoding possible in order for the model to be "scientific" forces a choice. As soon as we acknowledge that complex reality can and indeed must be encoded into distinctly different formal systems, the illusion of objectivity 
is destroyed. Lakoff uses the sentence "Don't think of an elephant" to illustrate the impossibility of escaping certain thoughts once such a framing event has occurred [9]. Modern science is framed by reductionist thought and this framing is so effective that the illusion of objectivity can do very well. A large part of Robert Rosen's work is devoted to helping us see the predicament we are in. It is very interesting how different people react to these ideas. If the concept of complexity being used here is not accepted by a person, there is almost no chance that the person will understand why objectivity is not ever possible. The irony is that that position is a subjective choice.

If this is understood, it becomes a simple matter to understand why thermodynamic reasoning is only accepted when it has a mechanistic counterpart. The largest model must be able to encompass everything scientific. The idea that distinctly different formal systems must exist as valid models is unacceptable. Thus the "useful" ideas from thermodynamics are made to be derivable from mechanistic reasoning, namely statistical mechanics, and any other conclusion drawn independent of that mechanical base are suspect.

\subsection{Reductionism needs a particular kind of mathematics to accomplish its goals}

The formal systems reductionist science use are mathematical. Yet among the rich formalisms in the total mathematical repertoire, only analytical mathematics is able to fill the needs of the reductionist paradigm. Calculus and differential equations are the essence of the Newtonian approach and were indeed created for that use. Thermodynamics and Rosen's approach to complexity utilize another kind of mathematics. It is topology that enters as the formalism that provides the needed structure for encoding relationships in the real world. The analytical mathematicians also were forced to utilize topology in the breakthroughs that occurred when the realization that so much of interest had to be encoded using non-linear differential equations as the equations of motion. Topology and differential geometry then were found useful even to reductionists. Out of this the nature of chaotic systems was born and chaos theory had a central role during a brief period of the evolution of complexity theory. It is still not clear to everyone that chaotic dynamics are part of the Newtonian paradigm and do not constitute a distinct formalism different from its largest model. The topological mathematics used to model complex systems and the topological mathe- 
matics that must be used to arrive at certain unique new conclusions about the real world using thermodynamic reasoning are very closely related.

Rosen, as a student of Rashevsky recognized that topological mathematics was necessary to create the relational biology they both had envisioned as the approach to living systems that was not locked to the severe limits of the reductionist paradigm. The elusive qualities of living things that distinguished them from non-living mechanistic things could only be dealt with by encodings into topological mathematical formalisms. Hence Rosen developed the metabolism-repair M, R system as the formalism he would manipulate to come up with a clear distinction between the classes of things we call "organisms" and the class of things we call "machines". In doing this he forced his audience to make a hard choice. Either they would accept a formalism that "kept the organization" but left out the "physics" or they were locked into the reductionist's world of physics which necessarily lost crucial aspects of the complex whole as it was reduced to mere material parts.

Thermodynamics in its most powerful form mimics this situation. There are many examples, but only a few need be mentioned to make this point.

\subsection{Topological reasoning in thermodynamics leads to powerful results}

One of the most interesting examples of the comparison between reductionist methods and relational or topological methods is in the proof of the second law of thermodynamics which was accomplished by Caratheodry after a discussion about the proofs existing up to then with Max Planck [8]. The existing proofs were done using the cyclic quasi-stationary processes developed by Carnot. Plank found these a very unsatisfying way to prove something having the global consequences of the second law. Caratheodry was able to construct a topological proof that rested on one piece of empirical input to confirm it. The second law had its global validity established by Joule's experiment for it was only necessary to give one instance of irreversibility in the real world to make the model valid.

The discovery of a metric structure for thermodynamics had been forsaken until Network Thermodynamics in the hands of Puesner revealed that the network thermodynamic model of a system was canonical. This discovery rests on the topological embedding of the system posited by Onsager, Prigogine and the others in a higher dimensional space[8]. 


\section{Will science extend to the modeling of complex reality or will it be restricted to the limited domain of the largest model formalism it clings to?}

This is a question that should not have to be asked. It is the self-imposed limits that scientists have used to frame the answer to the question "What is science?" that make it necessary. The framing of the question in this manner is strengthened by the enormous progress being made in technology. The hunger for more and more practical results makes it very difficult to persuade scientists to spend time and resources pursuing an approach that is just beginning to be recognized by significant minority among them. Should bright students be encouraged to work in this new area? Is it wise to devote any significant portion of the scarce resources given to science to the development of these radically new ideas? It seems to be easier for many scientists to choose an alternative definition for the word "complexity" and to make sure that this definition never allows those using it to step outside the reductionist paradigm.

There are a few who will not be restricted in that way. Two examples of recent progress in complexity science are very different in their area of application yet they hold in common their dedication to the new challenge to find distinct formal systems that can not be reduced to the Newtonian paradigm.

In their book Into the cool [10] using earlier work by Schneider and Kay [11] develop a thesis about the synergy between life on this planet and the conditions that sustain that life based on a broader interpretation of the second law of thermodynamics than used before. They see the second law as demanding that systems respond to imposed gradients by some form of self-organization in order to decrease the gradient. The alternative is to have enormous driving forces stressing the system. Hence the biosphere is believed to be the result of the tendency for the sun's energy to create stressing gradients. They present evidence for the role that life forms, weather patterns, etc. play in keeping the system stable. Clearly, a concept like this one is not going to be something we prove or disprove. At a time when so much controversy centers around the impact of human activity on planetary conditions, their endeavors do provide a model worth examining further before dismissing it out of hand merely because it forces us to abandon the restricted universe of discourse reductionist science has forced upon us.

The second area where some bold new ideas are being offered ins in 
the domain of what we often call the "mind-body" problem. The work of Bach-y-Rita as interpreted by Kercel $[12,13]$ and others goes tom the heart of many modern controversies. Their interpretation of real experiments involving the sensory behavior of humans is shaking quite a few foundations. At the center of all their models is the central role played by closed loops of causality. These loops are the self-referential heart of complex reality and the human mind seems replete with them. This work and he work of Louie [14], a student of Rosen, showing that Rosen's $\{M, R$ \} systems necessarily have non-computable aspects suggests that "artificial intelligence" and "artificial life" are indeed merely machine intelligence and simulations of systems that have some limited life-like qualities. These technological marvels are distinctly different from human intelligence and living systems in many import ways.

These new investigations as well as the areas of thermodynamics that have been largely disregarded because of their distinctly non-mechanistic character strongly suggest that science will find a way to include other formalisms and break free from the restrictions it has imposed in itself.

\section{References}

1. Rosen, R. Anticipatory Systems, New York, Pergamon ,1985

2. Rosen, R. LifeIitself, New York, Columbia, 1991

3. Rosen, R. Essays on LifeIitself, New York, Columbia, 2000

4. Mikulecky, D. C. The circle that never ends: Can Complexity be made simple? In Complexity in Chemistry, Biology, and Ecology, D. Bonchev and D. H. Rouvray, eds. New York, Springer, 2005.

5. Truesdell, C. Rational Thermodynamics, New York, McGraw-Hill, 1969

6. Onsager, L. Reciprocal Relations in Irreversible Processes I, Phys. Rev., 1931a, 37, 405-426.

7. Onsager, L. Reciprocal Relations in Irreversible Processes II, Phys. Rev., 1931b, 38, 2265-2279.

8. Mikulecky, D. C. Applications of Network Thermodynamics to Problems in Biomedical Engineering, New York, New York University Press, 1993.

9. Lakoff, G, Don't Think of an Elephant! Know Your Values and Frame the Debate White River Junction, Vermont, Chelsea Green Publishing, 2004

10. Schneider, E. D. and Sagan, D. Into the Cool, Chicago, University of Chicago, 2005

11. Schneider, E. D. and J. J. Kay, Life as a manifestation of the second law of thermodynamics, in Modeling Complex Biological Systems, M. Witten and D. C. Mikulecky, eds, Special Issue of Mathematical and Computer modeling, 1994, 19, 25-48.

12. Kercel, S. W. Journal of Integrative Neuroscience, 2005, 4, 403-406.

13. Kercel, S. W., Reber, A. S. and Manges, W. W. Some Radical Implications 
of Bach-y-Rita's Discoveries, Journal of Integrative Neuroscience, 2005, 4, 551-566.

14. Louie, A. H. Any Material Realization of the (M,R)-Systems Must Have Noncomputable Models, Journal of Integrative Neuroscience, 2005, 4, 423436. 